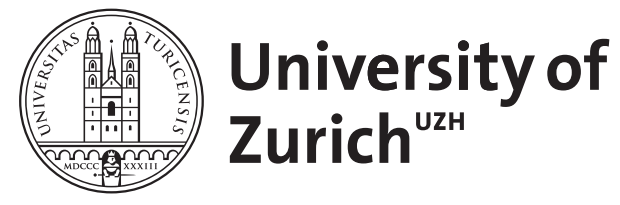

\title{
Raman spectra from ab initio molecular dynamics and its application to liquid S-methyloxirane
}

\author{
Luber, Sandra ; Iannuzzi, Marcella ; Hutter, Jürg
}

DOI: https://doi.org/10.1063/1.4894425

Posted at the Zurich Open Repository and Archive, University of Zurich ZORA URL: https://doi.org/10.5167/uzh-100689

Journal Article

Published Version

Originally published at:

Luber, Sandra; Iannuzzi, Marcella; Hutter, Jürg (2014). Raman spectra from ab initio molecular dynamics and its application to liquid S-methyloxirane. Journal of Chemical Physics, 141(9):094503.

DOI: https://doi.org/10.1063/1.4894425 


\section{AD| $\begin{aligned} & \text { The Journal of } \\ & \text { Chemical Physics }\end{aligned}$}

\section{Raman spectra from ab initio molecular dynamics and its application to liquid S- methyloxirane}

Sandra Luber, Marcella lannuzzi, and Jürg Hutter

Citation: The Journal of Chemical Physics 141, 094503 (2014); doi: 10.1063/1.4894425

View online: http://dx.doi.org/10.1063/1.4894425

View Table of Contents: http://scitation.aip.org/content/aip/journal/jcp/141/9?ver=pdfcov

Published by the AIP Publishing

\section{Articles you may be interested in}

A prototypical ionic liquid explored by ab initio molecular dynamics and Raman spectroscopy

J. Chem. Phys. 139, 144309 (2013); 10.1063/1.4823824

Simulations of vibrational spectra from classical trajectories: Calibration with ab initio force fields

J. Chem. Phys. 127, 084502 (2007); 10.1063/1.2756837

Ab initio molecular dynamics: Propagating the density matrix with Gaussian orbitals. III. Comparison with

Born-Oppenheimer dynamics

J. Chem. Phys. 117, 8694 (2002); 10.1063/1.1514582

Ab initio molecular dynamics: Propagating the density matrix with Gaussian orbitals. II. Generalizations based on mass-weighting, idempotency, energy conservation and choice of initial conditions

J. Chem. Phys. 115, 10291 (2001); 10.1063/1.1416876

Ab initio molecular dynamics: Propagating the density matrix with Gaussian orbitals

J. Chem. Phys. 114, 9758 (2001); 10.1063/1.1372182

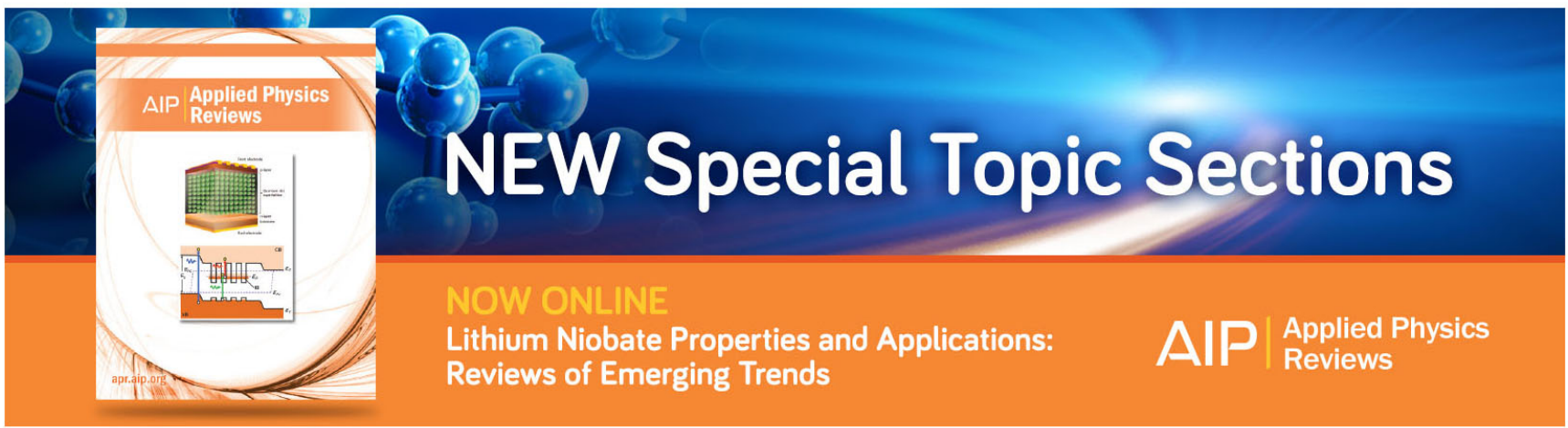




\title{
Raman spectra from ab initio molecular dynamics and its application to liquid S-methyloxirane
}

\author{
Sandra Luber, ${ }^{\text {a) }}$ Marcella lannuzzi, and Jürg Hutter \\ Department of Chemistry, University of Zurich, Winterthurerstrasse 190, 8057 Zurich, Switzerland
}

(Received 21 June 2014; accepted 20 August 2014; published online 4 September 2014)

\begin{abstract}
We describe the calculation of Raman spectra for periodic systems via ab initio molecular dynamics (AIMD) utilizing the Gaussian and plane wave method in the program package CP2K. The electricdipole-electric-dipole polarizability tensor has been implemented for an arbitrary shape of the simulation cell. In addition, a computationally efficient approach for its decomposition into local contributions is presented. As an example for the application of computational Raman spectroscopy to liquids, the Raman spectra of $S$-methyloxirane in the liquid phase have been calculated together with Raman spectra obtained from static calculations employing the double-harmonic approximation. The comparison to experimental data illustrates that a very good agreement between experiment and simulated spectra can be obtained employing AIMD, which takes into account anharmonicities and dynamical effects at ambient conditions. (C) 2014 AIP Publishing LLC. [http://dx.doi.org/10.1063/1.4894425]
\end{abstract}

\section{INTRODUCTION}

Methyloxirane is a colorless liquid produced on large scale industrially. It is commonly employed in the synthesis of polymers as well as an intermediate for a variety of compounds ranging from adhesives and coatings over solvents for food and cosmetics. Despite its wide use, it has to be handled with care since it may, among others, cause cancer and genetic defects. In this regard, analytical chemistry plays an important role, not only for the analysis of pure methyloxirane but also for the investigation of substances that may contain methyloxirane after the preparation process.

A standard technique in analytics is the measurement of Raman spectra. This gives valuable information about the system under study and can be used for the identification of molecular fingerprints. Although there are certain rules of thumb for the assignment of bands in the spectra, calculations are of great help for the interpretation of spectra as well as the detailed deduction of molecular structures and interactions between or even within molecules. The routine way for the calculation of Raman spectra within the BornOppenheimer approximation is based on the double-harmonic approximation $^{1,2}$ (in this work, we deal with off-resonance Raman spectra) starting with a geometry optimization of the system of interest (usually in the gas phase and assuming a temperature of $0 \mathrm{~K}$ ) in order to obtain its structure at the minimum of the electronic energy. Molecular vibrations described by normal modes can be obtained from the second derivatives of the electronic energy with respect to nuclear coordinates. The corresponding Raman intensities are evaluated as derivatives of the electric-dipole-electric-dipole polarizability tensor elements with respect to normal coordinates ${ }^{3}$ (for sake of brevity, we will refer to the electric-dipole-electric-dipole polarizability simply as polarizability in the following).

\footnotetext{
a)E-mail: sandra.luber@chem.uzh.ch
}

Despite several advantages, like being quite simple and computationally efficient, the double-harmonic approximation has also shortcomings. Aside from the fact that vibrational anharmonicities are not included, it is based on an entirely static picture. The effect of the temperature is only considered, if at all, a posteriori via the correction with Boltzmann population factors. ${ }^{4}$ This makes the adequate description of, e.g., solvent effects not straightforward. Continuum solvation models can be used to mimic the solvent environment, but it is difficult to accurately include specific interactions, such as hydrogen bonds, in this way. Another possibility is the combination of continuum solvation models with micro-solvation including explicit solvent molecules. Although this approach improves the agreement between computational and experimental data, ${ }^{5-8}$ it does not account for dynamic effects. This problem can, to some extend, be overcome by taking molecular structures from snapshots of molecular dynamics (MD) runs. ${ }^{6,9-12}$ Subsequent Raman spectra calculations for each of these molecular structures and averaging over the resulting spectra ${ }^{6,11,12}$ lead to a better agreement with experimental data, but — although still relying on the double-harmonic approximation — with a significantly higher computational effort compared to a single static harmonic calculation. Employing such a procedure, snapshots from $a b$ initio MD (AIMD) $)^{13,14}$ simulations have been found to be superior to the ones generated employing classical MD. ${ }^{6}$

A completely different approach solely based on AIMD is the computation of Raman spectra via time correlation functions of the polarizability tensor. This is not only a convenient way to simulate conformational dynamics and solvent effects at finite temperatures, the employment of periodic boundary conditions makes the calculation of Raman spectra for condensed matter possible. This approach has, for example, been used by Putrino and Parrinello ${ }^{15}$ for the calculation of Raman spectra of high-pressure ice and by Schettino et al. ${ }^{16}$ for a napthalene crystal. As the position operator is illdefined in periodic systems, the Berry-phase formulation for 


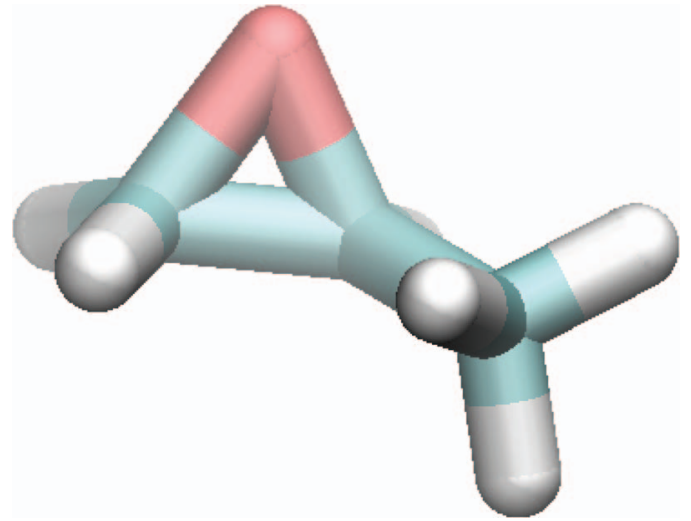

FIG. 1. Molecular structure of $S$-methyloxirane.

the electric polarization ${ }^{17-19}$ has been employed in the definition of the polarizability. Very recently, Gygi et al. ${ }^{20}$ presented Raman spectra of liquid water obtained from AIMD with a similar technique. In this case, the problem of the ill-defined position operator has been circumvented by using the velocity operator, which is well-defined in periodic systems. Within this formulation, the solution of an additional set of linear equations is necessary. Another procedure has been taken by Kirchner et al. ${ }^{21}$ employing maximally localized Wannier functions. ${ }^{22-25}$ In this way, the Raman spectra have been computed via molecular polarizabilities (see also Ref. 26) as derived from the variation of molecular electric dipoles when an external electric field is applied. ${ }^{21}$ This approach utilizes a finite difference scheme.

In this paper, we present Raman spectra of liquid $S$ methyloxirane (for its molecular structure, see Fig. 1) calculated with different approaches, which either rely on the static double-harmonic approximation or on AIMD. For the latter, the calculation of the polarizability tensor has been implemented into the program package $\mathrm{CP} 2 \mathrm{~K}$ using computationally efficient density functional perturbation theory (DFPT) ${ }^{27-31}$ based on the Berry-phase scheme for electric polarization in combination with the Gaussian and plane wave method. ${ }^{32}$ Furthermore, a new approach for the calculation of local polarizabilities is presented, which is possible at negligible additional cost during the calculation. The computational results are compared to experimental data of liquid $S$ methyloxirane.

The paper is organized as follows: after the theoretical background in Sec. II, the computational methodology is described in Sec. III. The influence of simulation parameters on the via AIMD computed Raman spectra of $S$-methyloxirane is discussed in Sec. IV. A comparison of these Raman spectra to experimental data and further analysis in terms of intraand intermolecular contributions can be found in Secs. V and VI, respectively. Static calculations are presented in Sec. VII, followed by a summary and outlook in Sec. VIII.

\section{THEORETICAL BACKGROUND}

Employing the Born-Oppenheimer approximation and Placzek's polarizability theory, ${ }^{33}$ the Raman intensity for the depolarized experimental set-up in the far from resonance ap- proximation (i.e., the incident light is far away from any resonance with an electronic transition) can be written as 3,34

$$
I_{\text {depol }} \sim 12 \gamma^{2}
$$

with the anisotropic Raman invariant (summation over Greek indices is implied)

$$
\begin{aligned}
\gamma^{2}= & \frac{1}{2}\left(3 \alpha_{\alpha \beta} \alpha_{\alpha \beta}-\alpha_{\alpha \alpha} \alpha_{\beta \beta}\right) \\
= & \frac{1}{2}\left[\left(\alpha_{x x}-\alpha_{y y}\right)^{2}+\left(\alpha_{x x}-\alpha_{z z}\right)^{2}+\left(\alpha_{y y}-\alpha_{z z}\right)^{2}\right. \\
& \left.+6\left(\alpha_{x y}^{2}+\alpha_{x z}^{2}+\alpha_{y z}^{2}\right)\right] .
\end{aligned}
$$

Here $\alpha_{\alpha \beta}$ is the $\alpha \beta$ component (the Greek subscripts $\alpha$ and $\beta$ represent the spatial $\mathrm{x}-, \mathrm{y}-$, and $\mathrm{z}$-coordinates) of the polarizability tensor $\boldsymbol{\alpha}$. For non-metallic systems, this component is given in the static limit $\omega_{L} \rightarrow 0\left(\omega_{L}\right.$ is the angular frequency of the incident light) as ${ }^{3}$

$$
\alpha_{\alpha \beta}=\frac{2}{\hbar} \sum_{j \neq 0} \frac{\left\langle\Phi_{0}\left|\hat{p}_{\alpha}\right| \Phi_{j}\right\rangle\left\langle\Phi_{j}\left|\hat{p}_{\beta}\right| \Phi_{0}\right\rangle}{\omega_{j}-\omega_{0}},
$$

where $\hbar\left(\omega_{j}-\omega_{0}\right)$ is the energy difference between the $j$ th excited electronic state and the ground state. The corresponding (N-particle) electronic wavefunctions are denoted as $\left|\Phi_{j}\right\rangle$ and $\left|\Phi_{0}\right\rangle$, respectively. $\hat{p}_{\alpha}$ is the $\alpha$ component of the (electronic) electric-dipole operator given as $\hat{p}_{\alpha}=-e \hat{r}_{\alpha}=-e \sum_{i=1}^{N} r_{\alpha, i}$ with $N$ being the number of electrons, $e$ the elementary charge, and $r_{\alpha, i}$ the $\alpha$ component of the position operator of electron $i$.

In case of periodic systems, the position operator is not uniquely defined. Going to the one-dimensional case and taking the electronic ground-state wavefunction $\left|\Phi_{0}\right\rangle$ to be periodic $\left(\left|\Phi_{0}\left(r_{\alpha}\right)\right\rangle=\left|\Phi_{0}\left(r_{\alpha}+L\right)\right\rangle\right.$ with $L$ being the imposed periodicity), this difficulty can be overcome by using the Berry-phase scheme $e^{17-19}$ where the expectation value for the position operator is evaluated as ${ }^{35}$

$$
\left\langle\Phi_{0}\left|\hat{r}_{\alpha}\right| \Phi_{0}\right\rangle=\frac{L}{2 \pi} \operatorname{Im} \ln \left\langle\Phi_{0}\left|\exp \left\{\mathrm{i} \frac{2 \pi}{L} r_{\alpha}\right\}\right| \Phi_{0}\right\rangle .
$$

A good approximation in AIMD simulations with a large simulation cell is to take the $\Gamma$ point of the Brillouin zone only. Moreover, in Kohn-Sham (KS) DFT ${ }^{36-43}$ the groundstate electronic wavefunction $\left|\Phi_{0}\right\rangle$ can be written as a Slater determinant of occupied ground-state molecular KS orbitals $\left|\psi_{i}\right\rangle$ that we take here to be real without loss of generality at $\Gamma$. The electric dipole in the $\alpha$ direction then becomes

$$
p_{\alpha}^{\text {Berry }}=f_{\text {occ }} \frac{e L}{2 \pi} \operatorname{Im} \ln \operatorname{det} S_{\alpha}
$$

with the matrix elements

$$
S_{\alpha, i j}=\left\langle\psi_{i}\left|\exp \left\{-\mathrm{i} \frac{2 \pi}{L} r_{\alpha}\right\}\right| \psi_{j}\right\rangle .
$$

Here $\left|\psi_{i}\right\rangle$ and $\left|\psi_{j}\right\rangle$ have the same occupation number $f_{\text {occ }}$, that is, one in the spin-polarized case and two in the spin-restricted case.

In the most general case, the simulation cell is described by three primitive lattice vectors $\boldsymbol{a}_{1}, \boldsymbol{a}_{2}$, and $\boldsymbol{a}_{3}$ forming a $3 \times 3$ matrix $\boldsymbol{h}=\left[\boldsymbol{a}_{1}, \boldsymbol{a}_{2}, \boldsymbol{a}_{3}\right]$ with elements $h_{\alpha \nu}=a_{\alpha \nu}$, 
$\alpha \in x, y, z, v \in 1,2,3$, and $\boldsymbol{h}_{\alpha}=\left(a_{\alpha 1}, a_{\alpha 2}, a_{\alpha 3}\right)$. The volume of the simulation cell is det $\boldsymbol{h}$. We work in scaled coordinates $\boldsymbol{s}=\boldsymbol{h}^{-1} \boldsymbol{r}^{16}$ in order to describe the electric polarization for the three-dimensional case, irrespective of the shape of the supercell. Thereby, the electric dipole is given as

$$
\boldsymbol{p}^{\text {Berry }}=f_{\text {occ }} \frac{e}{2 \pi} \boldsymbol{h} \operatorname{Im} \ln \operatorname{det} \boldsymbol{S},
$$

where the redefined $S_{\alpha}$ component has the matrix elements

$$
S_{\alpha, i j}=\left\langle\psi_{i}\left|\exp \left\{-\mathrm{i} 2 \pi \boldsymbol{h}_{\alpha}^{-1} \cdot \boldsymbol{r}\right\}\right| \psi_{j}\right\rangle .
$$

Employing Eq. (6), the polarizability can be evaluated in the electric-dipole approximation via $\alpha_{\alpha \beta}=-\delta p_{\alpha}^{\text {Berry }} / \delta E_{\beta}$, with $E_{\beta}$ being the external electric field in direction $\beta$.

In the framework of DFPT, ${ }^{27,28}$ the external electric field $\boldsymbol{E}$ can be treated as a perturbative parameter $\lambda$ coupled to the electric dipole $\boldsymbol{p}^{\text {Berry }}$. This gives rise to a perturbation $\lambda \epsilon^{(1)}$ $=-\boldsymbol{E} \cdot \boldsymbol{p}^{\text {Berry }}=-\sum_{\beta=1}^{3} E_{\beta} p_{\beta}^{\text {Berry }}$ added to the unperturbed KS energy functional $\epsilon^{(0)}{ }^{29}$ The total energy functional for the perturbation in $\beta$ direction amounts to

$$
\begin{aligned}
\epsilon= & \epsilon^{(0)}\left[\left\{\left|\psi_{i}^{(0)}\right\rangle-E_{\beta}\left|\psi_{i}^{(1), \beta}\right\rangle+\ldots\right\}\right] \\
& -E_{\beta} \epsilon^{(1), \beta}\left[\left\{\left|\psi_{i}^{(0)}\right\rangle-E_{\beta}\left|\psi_{i}^{(1), \beta}\right\rangle+\ldots\right\}\right]
\end{aligned}
$$

with the unperturbed and first-order perturbed KS orbitals $\left\{\left|\psi_{i}^{(0)}\right\rangle\right\}$ and $\left\{\left|\psi_{i}^{(1), \beta}\right\rangle\right\}$, respectively. The corresponding charge density up to first order in $E_{\beta}$ reads

$$
\begin{gathered}
n(\boldsymbol{r})=n^{(0)}(\boldsymbol{r})-E_{\beta} n^{(1), \beta}(\boldsymbol{r}), \\
n^{(1), \beta}(\boldsymbol{r})=\sum_{i} f_{\mathrm{occ}}\left[\psi_{i}^{(0) *}(\boldsymbol{r}) \psi_{i}^{(1), \beta}(\boldsymbol{r})+\psi_{i}^{(1), \beta *}(\boldsymbol{r}) \psi_{i}^{(0)}(\boldsymbol{r})\right],
\end{gathered}
$$

where we explicitly denote the dependence on the spatial coordinates.

Employing the orthogonality condition

$$
\left\langle\psi_{i}^{(0)} \mid \psi_{j}^{(1), \beta}\right\rangle=0 \quad \forall i, j
$$

the first-order perturbed KS orbitals $\left\{\left|\psi_{i}^{(1), \beta}\right\rangle\right\}$ are calculated via the inhomogeneous set of coupled equations

$$
\begin{aligned}
& -\sum_{j}\left(H^{(0)} \delta_{i j}-\left\langle\psi_{i}^{(0)}\left|H^{(0)}\right| \psi_{j}^{(0)}\right\rangle\right)\left|\psi_{j}^{(1), \beta}\right\rangle \\
& =P\left(\left.\int \frac{\partial^{2} E_{\mathrm{Hxc}}[n(\boldsymbol{r})]}{\partial n(\boldsymbol{r}) \partial n\left(\boldsymbol{r}^{\prime}\right)}\right|_{n^{(0)}(\boldsymbol{r})} n^{(1), \beta}\left(\boldsymbol{r}^{\prime}\right) d \boldsymbol{r}^{\prime}\left|\psi_{i}^{(0)}\right\rangle\right. \\
& \left.\quad+e \operatorname{Im} \frac{\partial}{\partial\left\langle\psi_{i}^{(0)}\right|} \ln \operatorname{det} \boldsymbol{S}_{\beta}\right)
\end{aligned}
$$

with

$$
\begin{aligned}
\frac{\partial}{\partial\left\langle\psi_{i}^{(0)}\right|} \ln \operatorname{det} \boldsymbol{S}_{\beta} & =\operatorname{Tr}\left[\boldsymbol{S}_{\beta}^{-1} \frac{\partial \boldsymbol{S}_{\beta}}{\partial\left\langle\psi_{i}^{(0)}\right|}\right] \\
& =\sum_{l}\left(S^{-1}\right)_{\beta, l i} \exp \left\{-\mathrm{i} 2 \pi \boldsymbol{h}_{\beta}^{-1} \cdot \boldsymbol{r}\right\}\left|\psi_{l}\right\rangle
\end{aligned}
$$

and the projector

$$
P=1-\sum_{i}\left|\psi_{i}^{(0)}\right\rangle\left\langle\psi_{i}^{(0)}\right| .
$$

$H^{(0)}$ is the unperturbed ground-state $\mathrm{KS}$ Hamiltonian and $E_{\mathrm{Hxc}}[n(\boldsymbol{r})]$ the Hartree, exchange, and correlation energy functional.

The polarizability is obtained as

$$
\boldsymbol{\alpha}=2 f_{\mathrm{occ}} \frac{e}{(2 \pi)^{2}} \boldsymbol{h} \operatorname{Im} \operatorname{Tr}\left[\boldsymbol{S}^{(1)}\right] \boldsymbol{h}^{T}
$$

with the matrix elements

$$
\left(S^{(1)}\right)_{\alpha \beta, i j}=\left\langle\psi_{i}^{(1), \alpha}\left|\exp \left\{-\mathrm{i} 2 \pi \boldsymbol{h}_{\beta}^{-1} \cdot \boldsymbol{r}\right\}\right| \psi_{j}^{(0)}\right\rangle\left(S^{-1}\right)_{\beta, j i} .
$$

In order to decompose the polarizability into local contributions, maximally localized Wannier functions can be employed. ${ }^{20,21,26}$ Based on the Gaussian type orbital method, we can take an alternative way avoiding computationally expensive localization procedures. Using atomic centered basis functions $\left\{\left|\chi_{\mu}\right\rangle\right\}$ and expansion coefficients $C_{i, v}$, KS orbitals are in general expressed as

$$
\left|\psi_{i}\right\rangle=\sum_{\mu} C_{i, \mu}\left|\chi_{\mu}\right\rangle .
$$

The $\alpha \beta$ component of the polarizability of a subset $A$ (which consists of at least one atom) can then be written as a sum of inter- and intra-subset contributions,

$$
\begin{aligned}
\alpha_{\alpha \beta}^{\mathrm{A}}= & \alpha_{\alpha \beta}^{\mathrm{A}, \text { intra }}+\alpha_{\alpha \beta}^{\mathrm{A}, \text { inter }} \\
= & 2 f_{\text {occ }} \frac{e}{(2 \pi)^{2}} \boldsymbol{h} \operatorname{Im} \operatorname{Tr}\left[\boldsymbol{S}_{\mathrm{A}, \text { intra }}^{(1)}\right] \boldsymbol{h}^{T} \\
& +2 f_{\mathrm{occ}} \frac{e}{(2 \pi)^{2}} \boldsymbol{h} \operatorname{Im} \operatorname{Tr}\left[\boldsymbol{S}_{\mathrm{A}, \text { inter }}^{(1)}\right] \boldsymbol{h}^{T}
\end{aligned}
$$

with

$$
\begin{aligned}
& \left(S_{\mathrm{A}, \text { intra }}^{(1)}\right)_{\alpha \beta, i j} \\
& =\left[\sum_{\mu \in A} \sum_{\nu \in A}\left(C_{i, \mu}^{(1), \alpha}\right)^{*}\left\langle\chi_{\mu}\left|\exp \left\{-\mathrm{i} 2 \pi \boldsymbol{h}_{\beta}^{-1} \cdot \boldsymbol{r}\right\}\right| \chi_{\nu}\right\rangle C_{j, \nu}^{(0)}\right]\left(S^{-1}\right)_{\beta, j i}, \\
& \left(S_{\mathrm{A}, \text { inter }}^{(1)}\right)_{\alpha \beta, i j} \\
& =\left[\sum_{\mu \in A} \sum_{\nu \notin A}\left(C_{i, \mu}^{(1), \alpha}\right)^{*}\left\langle\chi_{\mu}\left|\exp \left\{-\mathrm{i} 2 \pi \boldsymbol{h}_{\beta}^{-1} \cdot \boldsymbol{r}\right\}\right| \chi_{\nu}\right\rangle C_{j, \nu}^{(0)}\right]\left(S^{-1}\right)_{\beta, j i},
\end{aligned}
$$

and

$$
\alpha_{\alpha \beta}=\sum_{A} \alpha_{\alpha \beta}^{A}
$$

Here $C_{i, \mu}^{(1), \alpha}$ and $C_{i, v}^{(0)}$ are expansion coefficients corresponding to the (in $\alpha$-direction) first-order perturbed and unperturbed KS orbitals $\left\{\left|\psi_{i}^{(1), \alpha}\right\rangle\right\}$ and $\left\{\left|\psi_{i}^{(0)}\right\rangle\right\}$, respectively. In Eq. (19), we have distributed the contributions arising from 
the coupling of the atom(s) in subset A with the atom(s) not included in this subset in equal shares. This can, of course, be modified depending on the type of atoms/subsets and the goal of the calculation.

Along AIMD simulations, $\boldsymbol{\alpha}$ can be calculated as a function of time $t$ selecting snapshots of the trajectory at regular intervals. Vibrational spectra are obtained by Fourier transformation of certain time autocorrelation functions. ${ }^{44,45}$ The depolarized Raman intensity can be calculated from the autocorrelation of the polarizability components and subsequent Fourier transformation via

$$
\begin{aligned}
\gamma^{2}= & \frac{1}{2} \frac{1}{2 \pi}\left\{\int _ { - \infty } ^ { \infty } \mathrm { d } t \operatorname { e x p } \{ - \mathrm { i } \omega t \} \left\langle\left[\alpha_{x x}(0)-\alpha_{y y}(0)\right]\right.\right. \\
& \left.\times\left[\alpha_{x x}(t)-\alpha_{y y}(t)\right]\right\rangle \\
& +\int_{-\infty}^{\infty} \mathrm{d} t \exp \{-\mathrm{i} \omega t\}\left\langle\left[\alpha_{x x}(0)-\alpha_{z z}(0)\right]\left[\alpha_{x x}(t)-\alpha_{z z}(t)\right]\right\rangle \\
& +\int_{-\infty}^{\infty} \mathrm{d} t \exp \{-\mathrm{i} \omega t\}\left\langle\left[\alpha_{y y}(0)-\alpha_{z z}(0)\right]\left[\alpha_{y y}(t)-\alpha_{z z}(t)\right]\right\rangle \\
& +6\left[\int_{-\infty}^{\infty} \mathrm{d} t \exp \{-\mathrm{i} \omega t\}\left\langle\alpha_{x y}(0) \alpha_{x y}(t)\right\rangle\right. \\
& +\int_{-\infty}^{\infty} \mathrm{d} t \exp \{-\mathrm{i} \omega t\}\left\langle\alpha_{x z}(0) \alpha_{x z}(t)\right\rangle \\
& \left.\left.+\int_{-\infty}^{\infty} \mathrm{d} t \exp \{-\mathrm{i} \omega t\}\left\langle\alpha_{y z}(0) \alpha_{y z}(t)\right\rangle\right]\right\}
\end{aligned}
$$

The resulting intensities are multiplied by the harmonic approximation quantum correction factor $\frac{(\hbar \omega / k T)}{1-\exp (-\hbar \omega / k T)}(k$ : Boltzmann's constant, $T$ : temperature) that has been shown to satisfy both the detailed balance condition and the fluctuation-dissipation theorem (for details, see Ref. 46).

Employing Eqs. (17) and (21), the Raman spectra obtained for a simulation cell containing $N_{\text {mol }}$ subsets, which we take here to be molecules for the sake of brevity, can be analyzed in more detail. As a first step, an anisotropic Raman invariant $\gamma_{\text {tot,intra }}^{2}$ is computed by replacing the polarizability components $\alpha_{\alpha \beta}$ in Eq. (21) with $\alpha_{\alpha \beta}^{\text {tot,intra }}$. The latter is calculated as $\alpha_{\alpha \beta}^{\text {tot,intra }}=\sum_{i}^{N_{\text {mol }}} \alpha_{\alpha \beta}^{i, \text { intra }}$. Analogously, an intermolecular part $\gamma_{\text {tot,inter }}^{2}$ is obtained using $\alpha_{\alpha \beta}^{\text {tot,inter }}$, defined as $\alpha_{\alpha \beta}^{\text {tot,inter }}=\sum_{i}^{N_{\text {mol }}} \alpha_{\alpha \beta}^{i \text {,inter }}$, in Eq. (21) instead of $\alpha_{\alpha \beta}$.

As a second step, $\gamma_{\text {tot, intra }}^{2}$ can be further split into contributions considering individual molecules. This can be achieved by dividing the terms of the type

$$
\int_{-\infty}^{\infty} \mathrm{d} t \exp \{-\mathrm{i} \omega t\}\left\langle\sum_{A=1}^{N_{\text {mol }}} \alpha_{\alpha \beta}^{A, \text { intra }}(0) \sum_{B=1}^{N_{\text {mol }}} \alpha_{\alpha \beta}^{B, \text { intra }}(t)\right\rangle
$$

in the intensity expression [see Eq. (21)] into intra- and intermolecular contributions: the term with $A=B$ is employed to compute $\gamma_{A \text {,mol,intra }}^{2}$ leading to an intramolecular anisotropic Raman invariant $\gamma_{\text {mol,intra }}^{2}=\sum_{A} \gamma_{A \text {,mol,intra }}^{2}$, whereas all terms with $A \neq B$ are used for the evaluation of intermolecular contributions contained in $\gamma_{\text {mol,inter }}^{2}$. In a similar way, the contributions in $\gamma_{\text {tot,inter }}^{2}$ and the cross correlation spectrum between $\gamma_{\text {tot,intra }}^{2}$ and $\gamma_{\text {tot,inter }}^{2}$ can be decomposed.

\section{COMPUTATIONAL METHODOLOGY}

We have implemented the calculation of the polarizability for (non-)periodic systems into the CP2K program package $^{47}$ which was employed for the Born-Oppenheimer AIMD simulations presented in this paper. KS-DFT was used as the electronic structure method in the framework of the Gaussian and plane waves formalism. We tested three different density functionals: BP86, ${ }^{48,49}$ that has been found to perform well in static frequency calculations within the doubleharmonic approximation, ${ }^{50-63} \mathrm{BLYP},{ }^{48,64}$ a popular density functional for IR spectra calculations via AIMD (see, for example, Refs. 21 and 65-72), and $\mathrm{PBE}^{73,74}$ for comparing purposes. BLYP-Goedecker-Teter-Hutter (GTH) (in case of PBE the PBE-GTH) pseudopotentials ${ }^{75-77}$ and the TZVPGTH and DZVP-GTH basis sets were applied. Grimme's D3 dispersion corrections ${ }^{78}$ were added (except in the case of the BP86 density functional). For the condensed phase simulation of $20 \mathrm{~S}$-methyloxirane, we employed a cubic cell containing 20 molecules corresponding to a density of $0.830 \mathrm{~g} / 1$ at $298.5 \mathrm{~K} .{ }^{79}$ The propagation time step $(\delta t)$ in the AIMD simulation was set to $0.5 \mathrm{fs}$. If not mentioned otherwise, the polarizability tensor was calculated for each time step. Before the production runs in the NVE ensemble, the systems were equilibrated in the NVT ensemble for $5 \mathrm{ps}$. The temperature was set to $298.5 \mathrm{~K}$ via a Nosé-Hoover chain thermostat. ${ }^{80,81}$ The spectra for the depolarized experimental set-up (compare Sec. IV) were produced by a modified and extended version of the program TRAVIS ${ }^{21,82}$ applying zero padding and a Hanning type window function.

For the static calculations, the molecular structures were optimized employing the program package TURBOMOLE, ${ }^{83}$ version 6.4 , by means of KS-DFT. The Raman spectra were obtained with the program package MOVIPAC ${ }^{61}$ employing the electronic energy gradients and polarizability tensors obtained from TURBOMOLE. The second derivatives of the electronic energy with respect to the nuclear coordinates were calculated numerically by MOVIPAC for displaced structures via a 3-point central difference formula. ${ }^{84}$ The step length in the numerical differentiation was set to 0.01 bohr. Ahlrichs' def2-TZVP basis set ${ }^{85}$ was used together with the BLYP density functional. The resolution-of-the-identity density-fitting technique ${ }^{86-88}$ was exploited in all calculations. For the calculation using $20 \mathrm{~S}$-methyloxirane molecules, dispersion corrections ${ }^{78}$ were also been added in analogy to the AIMD calculation. The presented Raman spectra are plotted for the depolarized experimental set-up with a Lorentzian band width at half-maximum height of $15 \mathrm{~cm}^{-1}$.

\section{PARAMETER DEPENDENCE OF THE RAMAN SPECTRA}

$S$-methyloxirane is a well-known molecule and widely studied for the calculation of vibrational spectra (see, e.g., Refs. 34, 52, and 89-95). Nevertheless, to the best of our knowledge, no computational study of the Raman spectrum of its liquid phase has been published yet. In this paper, we present the Raman spectra of liquid $S$-methyloxirane computed with the protocol described in Secs. II and III. 

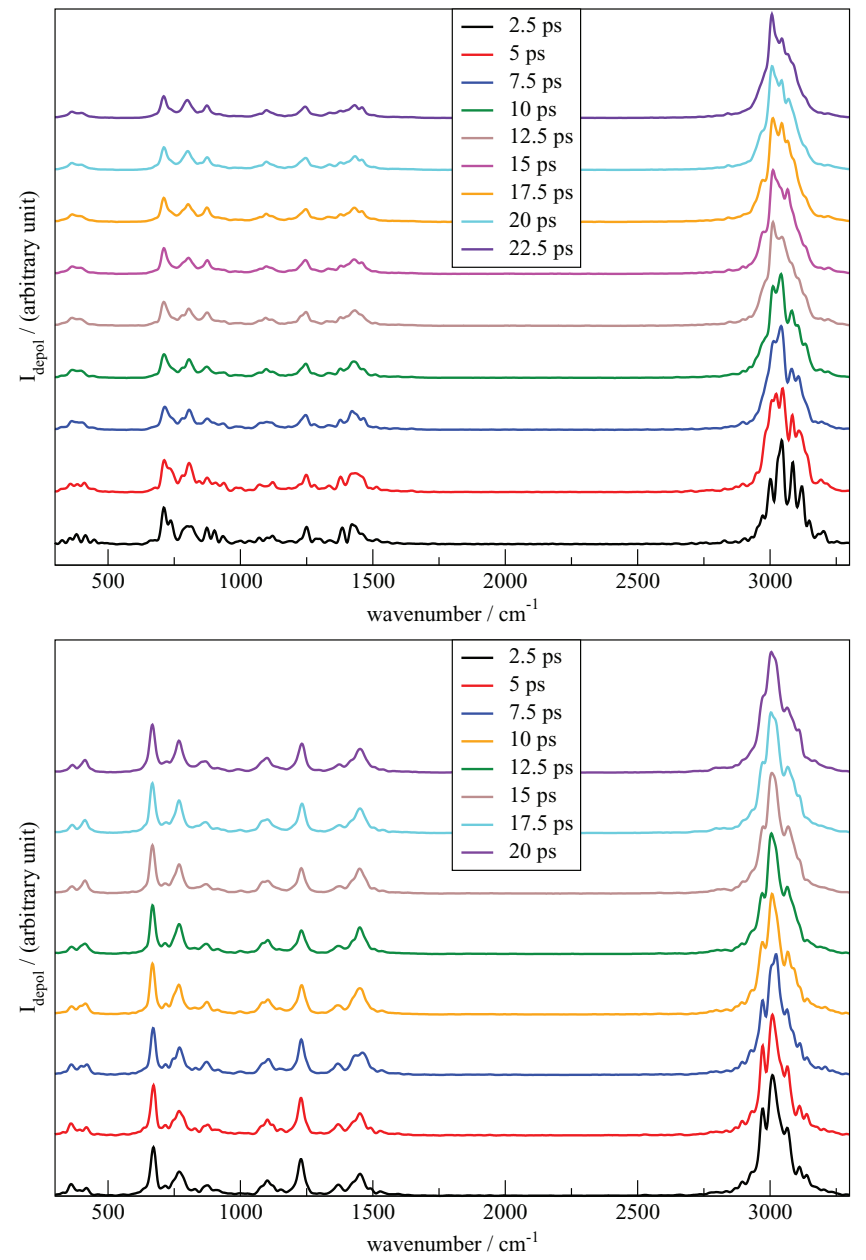

FIG. 2. Depolarized Raman spectra of liquid $S$-methyloxirane obtained from AIMD for different sampling times (top: BP86/TZVP-GTH; bottom: BLYP/TZVP-GTH/D3).

An important factor in the calculation of vibrational spectra from AIMD runs is the sampling of all accessible conformations of the system under study corresponding to the addressed state in the phase space. In order to guarantee the convergence of the sampling, the AIMD simulation has to be propagated for a sufficiently long time. Here, we assess the quality of the sampling by comparing spectra obtained from trajectories of different length. Fig. 2 shows Raman spectra of liquid $S$-methyloxirane obtained with the BP86 density functional for varying sampling times. Similar to the observations in Ref. 21, the most intense bands are already well recognizable after 5 ps. At 10 ps, a large part of the bands - especially for wavenumbers lower than $1600 \mathrm{~cm}^{-1}$ - is almost converged so that only tiny changes are observed for longer runs. After $12.5 \mathrm{ps}$, the wavenumber region below $1600 \mathrm{~cm}^{-1}$ is completely converged. For the $\mathrm{C}-\mathrm{H}$ stretching vibrations occurring above $2900 \mathrm{~cm}^{-1}$, small differences in the band intensities are still obtained up to $22.5 \mathrm{ps}$. In case of the BLYP density functional (see bottom of Fig. 2), the convergence behavior is comparable to the one obtained with BP86.

Another simulation parameter is the time step for the polarizability calculation along the trajectory $\left(\delta t_{\mathrm{pol}}\right)$. One can choose if the polarizability tensor is calculated at each propa- gation time step or less. The latter has the advantage that the linear response equations are not evaluated at every AIMD step, which leads to saving in computational time. Based on the $20 \mathrm{ps}$ long trajectory obtained with BLYP and a propagation time step of $\delta t=0.5 \mathrm{fs}$, we have observed that a value of $\delta t_{\mathrm{pol}} \leq 5.0 \mathrm{fs}$ gives rise to only minor changes in the Raman spectra, in particular for the lower wavenumber range till $2000 \mathrm{~cm}^{-1}$ [see Fig. 1 in the supplementary material ${ }^{96}$ ]. For $\delta t_{\text {pol }}>5.0 \mathrm{fs}$, we have observed a poor quality of the spectrum. Further tests also show that the Raman spectra for this specific system are mainly determined by the quality of the trajectory. Calculating the trajectory and Raman spectra with a double-zeta valence basis set with one set of polarization functions (DZVP-GTH) leads to remarkable changes (see Fig. 2 in the supplementary material $\left.{ }^{96}\right)$. In contrast, changing the basis set only for the linear response calculations does not significantly modify the results as long as the trajectory has been generated with BLYP/TZVP-GTH/D3 (see Fig. 2 in the supplementary material ${ }^{96}$ ). Similarly, negligible changes are obtained if the BLYP-GTH pseudopotential is replaced by the PBE-GTH pseudopotential or if the BLYP density functional is substituted by the BP86 or PBE density functional in the linear response calculation (compare Fig. 3 in the supplementary material ${ }^{96}$ ).

\section{COMPARISON TO EXPERIMENTAL DATA}

Fig. 3 displays the comparison of the computed Raman spectra for the 20 ps long trajectory with the experimental result reported in Ref. 34. The position of the doublet at around $380 \mathrm{~cm}^{-1}$ is well reproduced in the calculation. The relative intensity of the two bands is correctly given by the BP86 calculation, whereas the BLYP density functional shows the reverse order. The two most intense bands - mainly arising from $\mathrm{C}-\mathrm{O}$ and $\mathrm{C}-\mathrm{CH}_{2}$ stretching vibrations ${ }^{34}$ - occur in the measured spectrum at about 750 and $830 \mathrm{~cm}^{-1}$, at 711 and $800 \mathrm{~cm}^{-1}$ in the case of BP86, and at 686 and $768 \mathrm{~cm}^{-1}$ if BLYP is employed. An underestimation of experimental frequencies has already been observed in previous frequency

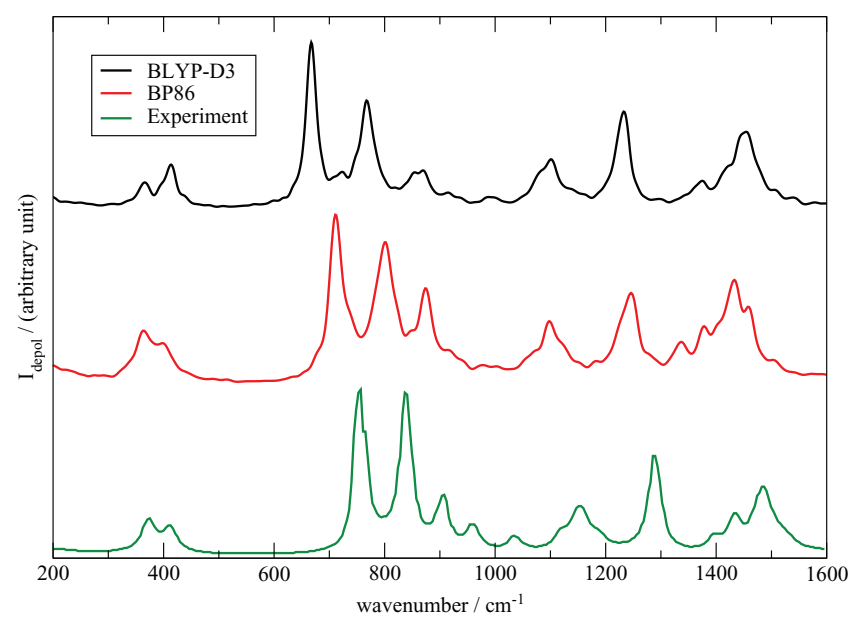

FIG. 3. Depolarized Raman spectra of liquid $S$-methyloxirane from AIMD (BP86/TZVP-GTH; BLYP/TZVP-GTH/D3) and the experimental spectrum (neat liquid) reproduced from Ref. 34 (for details, see text). 
calculations with AIMD (see, e.g., Refs. 68, 69, and 97). The relative intensity of the calculated bands at $800 \mathrm{~cm}^{-1}$ (with BP86) and $768 \mathrm{~cm}^{-1}$ (with BLYP) is somewhat lower than the one of the experimental band at $824 \mathrm{~cm}^{-1}$. Nevertheless, the agreement is very good, especially if compared to experimental data of $S$-methyloxirane vapor that show the most intense bands at around 960 and $1270 \mathrm{~cm}^{-1} \cdot 34,94$ Even a tiny broadening at the higher-wavenumber side of the experimental band at about $745 \mathrm{~cm}^{-1}$ is obtained for the corresponding band in the calculation employing BP86. With BLYP, this band occurs with a relatively small intensity between the two strong bands at 686 and $768 \mathrm{~cm}^{-1}$.

The radial distribution functions shown in Fig. 4 give further information about structural properties of the system under study. Their quite simple pattern illustrates that $S$-methyloxirane is an unstructured liquid. Solely minor differences in the $\mathrm{C}-\mathrm{H}$ radial distribution are observed in case of the two density functionals. The two first peaks at around 1.1 and $2.2 \AA$ are due to intramolecular distances while the peaks at about 2.8 and $3.5 \AA$ are a consequence of both inter- and intramolecular distances. The $\mathrm{C}-\mathrm{O}$ radial distributions show a first maximum at around $1.5 \AA$ resulting from the $\mathrm{C}-\mathrm{O}$ bonds of the oxirane ring. The distance from the ring oxygen to the carbon atom of the methyl group gives rise to the second maximum at about $2.5 \AA$. The position of the maxima agree very well. However, a small broadening at the longer distance side is observed for BLYP compared to the analogous band obtained with BP86. Contrary to that, the band occurring at around $3.5 \AA$, which corresponds to intermolecular $\mathrm{C}-\mathrm{O}$ distances, is broader at the lower distance side in the case of BLYP. These differences may also be caused by the D3 dispersion correction, which was only applied in the calculation with BLYP. A similar picture is found for the $\mathrm{C}-\mathrm{C}$ radial distributions, where the first maximum arises from the $\mathrm{C}-\mathrm{C}$ bonds and the second maximum from the distance of the methyl carbon atom with its second nearest carbon neighbor. This indicates that intramolecular $\mathrm{C}-\mathrm{C}$ and $\mathrm{C}-\mathrm{O}$ bonds in the BLYP calculation are partly longer compared to the ones evaluated with BP86. That observation can explain the shift of the bands in the BLYP spectrum, compared to the ones computed with BP86, in the wavenumber range from 650 to $850 \mathrm{~cm}^{-1}$. Indeed, these frequencies are related to stretching vibrations of the $\mathrm{C}-\mathrm{C}$ and $\mathrm{C}-\mathrm{O}$ bonds.

On the other hand, the $\mathrm{C}-\mathrm{H}_{2}$ twist motions responsible for the band at $869 \mathrm{~cm}^{-1}$ and $873 \mathrm{~cm}^{-1}$ in case of BLYP and BP86, respectively, do not involve significant changes of the $\mathrm{C}-\mathrm{C}$ and $\mathrm{C}-\mathrm{O}$ bonds.

The remaining computed bands up to $1600 \mathrm{~cm}^{-1}$ are also somewhat shifted to lower wavenumbers compared to the experimental data, but it is straightforward to assign the bands in the measured spectrum to the calculated ones. Also the evaluated relative intensities match the measured ones very well. For instance, the strong experimental band between 1200 and $1300 \mathrm{~cm}^{-1}$, which, according to static calculations, corresponds to $\mathrm{C}-\mathrm{H}$ bending and $\mathrm{C}-\mathrm{CH}_{2}$ stretching vibrations and is by far the most intense one in the experimental gas-phase spectrum of $S$-methyloxirane, ${ }^{34,94}$ is perfectly reproduced in the calculations. This is especially true in case of BP86, where the shift to lower wavenumbers is less pronounced than in
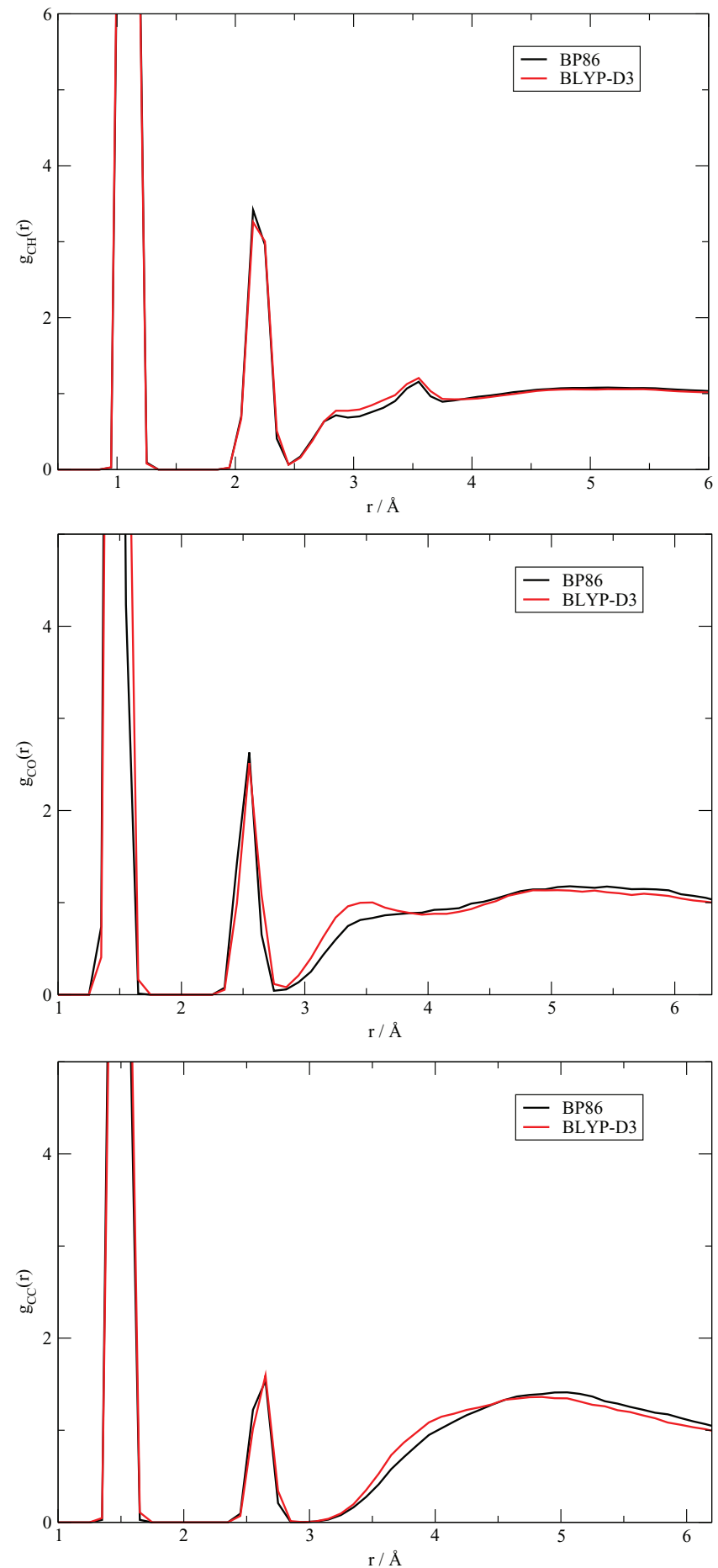

FIG. 4. $\mathrm{C}-\mathrm{H}$ (top), $\mathrm{C}-\mathrm{O}$ (middle), and $\mathrm{C}-\mathrm{C}$ (bottom) radial distribution functions obtained from the 20 ps long AIMD simulations (BP86/TZVP-GTH; BLYP/TZVP-GTH/D3).

case of BLYP. The smaller shift correlates well with the above observation of slightly longer $\mathrm{C}-\mathrm{C}$ bonds in the BLYP AIMD simulation. In addition, a good agreement can be observed for weaker bands. The band at around $1450 \mathrm{~cm}^{-1}$, predominantly arising from methyl asymmetric bending vibrations, ${ }^{34}$ is found with a similar relative intensity both in experiment and calculation, whereby the broad band is split into a doublet in case of BP86. Furthermore, the band measured at around $1140 \mathrm{~cm}^{-1}$ is computed with a wavenumber of about 
$1100 \mathrm{~cm}^{-1}$ and a broadening close to the one found in experiment. In this wavenumber range, $\mathrm{C}-\mathrm{H}_{2}$ wagging and $\mathrm{C}-$ $\mathrm{H}_{2} / \mathrm{C}-\mathrm{H}_{3}$ rocking motions occur. The evaluated wavenumbers are very similar for both the BLYP and BP86 calculations in agreement with the small differences found in the $\mathrm{C}-\mathrm{H}$ radial distribution (see Fig. 4).

\section{DECOMPOSITION OF RAMAN INTENSITIES: INTRA- AND INTERMOLECULAR CONTRIBUTIONS}

In order to trace back the origin of Raman bands in more detail, the Raman spectrum can be analyzed in terms of intraand intermolecular contributions (compare Sec. II). We performed such an analysis for the 20.0 ps long BLYP/TZVPGTH/D3 run where $\delta t_{\text {pol }}$ was set to $5.0 \mathrm{fs}$.

The corresponding Raman spectra are given in the upper part of Fig. 5. The intramolecular spectrum arising from $\gamma_{\text {tot,intra }}^{2}$ contributes by far the largest part to the overall Raman intensity (see spectrum labeled with "intra(tot)" in Fig. 5). The entirely intermolecular contribution from $\gamma_{\text {tot,inter }}^{2}$ is negligibly small (compare spectrum denoted as "inter(tot)" in Fig. 5). Noteworthy is the difference between the complete spectrum and the one obtained from $\boldsymbol{\alpha}^{\text {tot,intra }}$ at around 400 $\mathrm{cm}^{-1}$. This doublet is less intense in the full Raman spec-
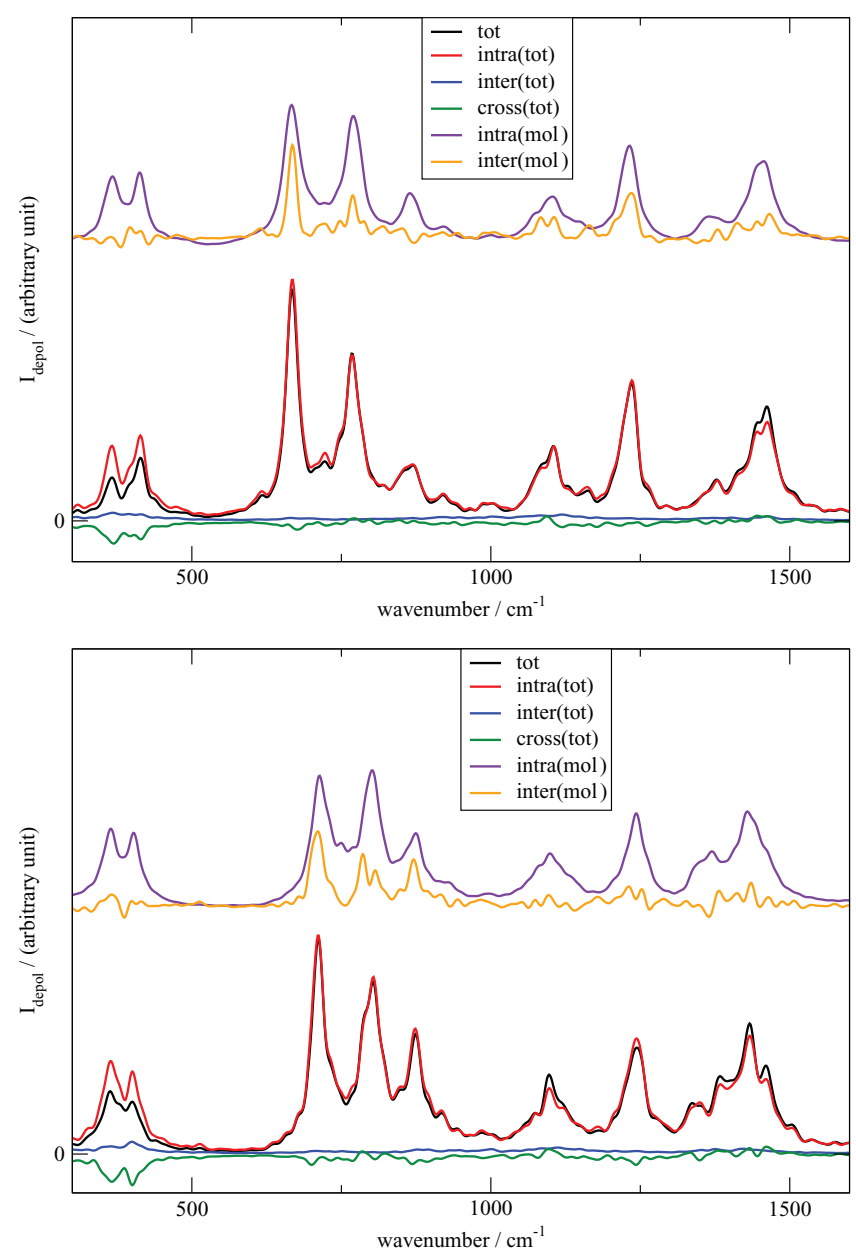

FIG. 5. Calculated depolarized Raman spectrum (top: BLYP/TZVPGTH/D3; bottom: BP86/TZVP-GTH) of liquid $S$-methyloxirane ("tot") decomposed into intra- and intermolecular contributions (for details, see text). trum than in the one evaluated from $\boldsymbol{\alpha}^{\text {tot,intra }}$. As illustrated in Fig. 5, the reason for this is the negative contribution arising from the time cross correlation between $\boldsymbol{\alpha}^{\text {tot,intra }}$ and $\boldsymbol{\alpha}^{\text {tot,inter }}$ (see spectrum named "cross(tot)" in Fig. 5).

Since $\boldsymbol{\alpha}^{\text {tot,inter }}$ gives only a minor contribution, we focus on the intramolecular part in the following. $\gamma_{\text {tot,intra }}^{2}$ can be further decomposed into $\gamma_{\text {mol,intra }}^{2}$ and $\gamma_{\text {mol,inter }}^{2}$ (see spectra "intra(mol)" and "inter(mol)" in Fig. 5, respectively) as derived in Sec. II. The bands originating from $\gamma_{\text {mol,intra }}^{2}$ are more intense than the ones resulting from $\gamma_{\text {mol,inter }}^{2}$. However, the intermolecular contribution is the main reason for the observed higher band intensity of the doublet at the higher wavenumber side at around $400 \mathrm{~cm}^{-1}$. The intermolecular component also primarily leads to the much higher intensity of the band at about $690 \mathrm{~cm}^{-1}$ compared to the following one around $770 \mathrm{~cm}^{-1}$. Smaller contributions of the intermolecular part are also found for the other bands in the spectrum.

A similar picture is obtained if the same analysis is performed for the 20 ps long BP86/TZVP-GTH simulation (see lower part of Fig. 5). The intermolecular contribution is responsible for the much higher intensity of the band around $700 \mathrm{~cm}^{-1}$ compared to the one at about $800 \mathrm{~cm}^{-1}$. These two bands are more or less equally intense in the intramolecular spectrum (see spectrum "intra(mol)" in Fig. 5), which is in very good agreement with the experimental spectrum. Especially, the band observed at around $1440 \mathrm{~cm}^{-1}$ in the experimental spectrum appears to be mainly originating from intermolecular contributions in the calculations (see in particular the spectra named "inter(mol)" in Fig. 5).

\section{COMPARISON TO STATIC CALCULATIONS}

A cluster of $20 S$-methyloxirane molecules, which was obtained from a snapshot of the AIMD run, has also been taken for a geometry optimization and subsequent standard Raman calculation within the double-harmonic approximation (assuming a temperature of $0 \mathrm{~K}$ ). As can be seen in Fig. 6, the intensities obtained from the AIMD and static calculation

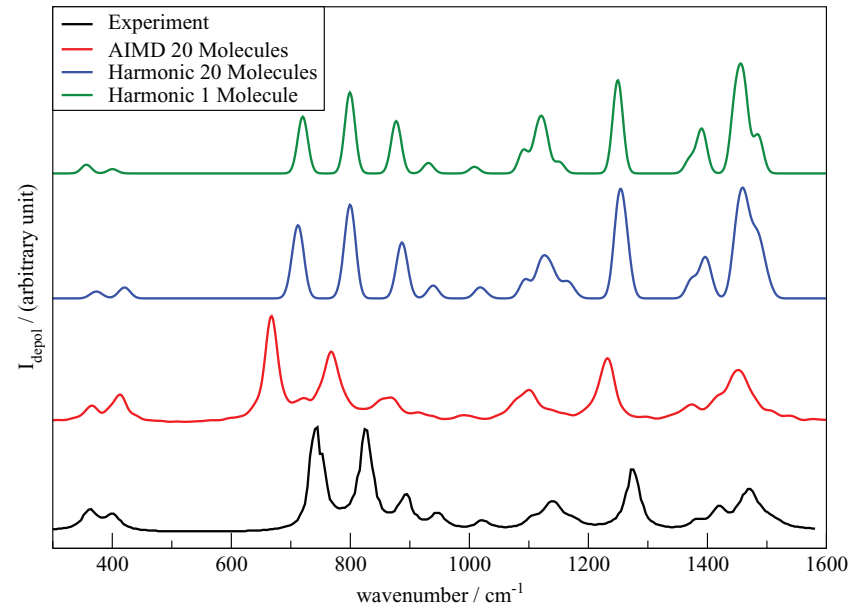

FIG. 6. Depolarized Raman spectra of $S$-methyloxirane obtained from AIMD (BLYP/TZVP-GTH/D3), from static calculations employing the double-harmonic approximation (BLYP/def2-TZVP/D3), and the experimental spectrum taken from Ref. 34 . 
are quite different. The most intense bands in the wavenumber range till $1600 \mathrm{~cm}^{-1}$ are observed in the static calculation at around 1250 and $1450 \mathrm{~cm}^{-1}$, which does neither agree with the AIMD spectrum nor with the experimental data for liquid $S$-methyloxirane. The two highest bands in the experimental spectrum around $800 \mathrm{~cm}^{-1}$ are also found in the static calculation with two quite intense bands at about 700 and 800 $\mathrm{cm}^{-1}$. The positions of the bands in the static calculation are in better agreement with experiment than the AIMD results. Some smaller differences between the experimental/AIMD spectrum and the static one are also visible, e.g., for the band in the range from about 1100 to $1200 \mathrm{~cm}^{-1}$. It is well known that BLYP in combination with the double-harmonic approximation leads to frequencies quite close to experimental fundamental ones. Inclusion of anharmonic corrections as done by the AIMD calculation thus apparently deteriorates the agreement with experimental data.

Using a smaller number of $S$-methyloxirane molecules in the static calculation does not lead to a significant change in the spectrum. This is obvious in Fig. 6 showing the static spectra obtained with only one $S$-methyloxirane molecule. The band around $1150 \mathrm{~cm}^{-1}$ is found with a slightly higher intensity and the bands at about $1450 \mathrm{~cm}^{-1}$ feature a somewhat different, less broadened band shape, which is, as expected, in worse agreement with the experimental data for liquid $S$ methyloxirane.

These findings show that the calculation within the double-harmonic approximation is not able to correctly reproduce the band shapes and intensities of the liquid as modeled by $20 S$-methyloxirane molecules (and a temperature of $0 \mathrm{~K}$ ). However, employing the same system in a periodic AIMD simulation gives rise to a spectrum in much better agreement with experimental data since, in addition to the periodicity of the system, the dynamics at ambient conditions is automatically taken into account.

\section{SUMMARY AND OUTLOOK}

We have described an efficient computational set-up for the calculation of polarizabilities and Raman spectra via AIMD utilizing the Gaussian and plane waves method implemented in the $\mathrm{CP} 2 \mathrm{~K}$ program package. The spectra have been evaluated via autocorrelation functions of the polarizability tensor that has been obtained via DFPT using the Berry-phase formulation for the electric polarization. This approach considers the dynamic behavior of the system under study and is thus ideally suited for the consideration of solvent effects in Raman spectra. It can furthermore straightforwardly be applied to liquids and solids under periodic boundary conditions with an arbitrary shape of the simulation cell. In addition to that, we have presented a new approach for the computation of local polarizabilities, which facilitates the interpretation of the calculated spectra and gives information about the system under study. This calculation does not require any localization procedure and comes at a negligible additional cost in the spectra calculation. The polarizability calculations can also be performed as post-processing of any trajectory file. This allows the separation of the trajectory and polarizability calculation and gives more flexibility regarding the computational parameters and effort.

As an example, we have presented the Raman spectra of liquid $S$-methyloxirane, a molecule widely used as solvent in industry. Employing a simulation cell containing $20 S$ methyloxirane molecules, already a simulation time of $7.5 \mathrm{ps}$ has been found to be enough to obtain a good description of the characteristic bands in the spectrum, especially for the bands below $2000 \mathrm{~cm}^{-1}$. Furthermore, we have demonstrated that it is not necessary to calculate the polarizability tensor at every time step for the 20 ps long trajectory, which leads to a significant reduction of computational cost. Investigation of the basis set and density functional dependence reveals that the appearance of the spectra is to a large extent influenced by the quality of the underlying trajectory, for which the property tensors are calculated. This finding suggests that the computational effort can additionally be reduced by choosing a tailored, computationally less demanding basis set for the polarizability calculation.

The comparison to the measured spectrum of liquid $S$ methyloxirane reveals a very good agreement between experimental and computational results, both in case of the BP86 and BLYP density functional. A slightly better match with the experimental frequencies in the wavenumber range from about 600 to $1500 \mathrm{~cm}^{-1}$ has been observed for BP86. A static calculation within the double-harmonic approximation leads to band positions, which are in general in closer accordance with experimental data. Nevertheless, the band intensities and band shapes do not resemble the experimental ones to a large extent. Analysis of the AIMD results in terms of inter- and intramolecular contributions further shows that the band shape of the doublet at around $400 \mathrm{~cm}^{-1}$ corresponding to skeletal vibrations is largely determined by intermolecular contributions and cross-correlation effects between intra- and intermolecular parts. Moreover, the different intensities of the two most intense bands in the calculated spectra are mainly a result of a large positive intermolecular contribution to the band around $700 \mathrm{~cm}^{-1}$. In the pure intramolecular spectra, these bands have more or less equal intensity.

This makes clear that AIMD is an excellent approach for the calculation of vibrational spectra of liquids. Since dynamic effects at ambient conditions are considered in the AIMD simulation, a very good agreement with experimental data can be achieved. An even higher agreement with experiment may be obtained for hybrid density functionals such as $\mathrm{PBE}^{98}$ that has been found to be superior to non-hybrid density functionals in case of IR spectra calculated from AIMD. ${ }^{99}$

\section{ACKNOWLEDGMENTS}

We acknowledge generous computing resources from the Swiss National Supercomputing Center (Project No. S425).

${ }^{1}$ E. B. Wilson, Jr., J. C. Decius, and P. C. Cross, Molecular Vibrations (McGraw-Hill, New York, 1955).

${ }^{2}$ J. Neugebauer, M. Reiher, C. Kind, and B. A. Hess, J. Comput. Chem. 23, 895 (2002).

${ }^{3}$ D. A. Long, The Raman Effect: A Unified Treatment of the Theory of Raman Scattering by Molecules (John Wiley \& Sons, New York, 2002).

${ }^{4}$ M.-P. Gaigeot, Phys. Chem. Chem. Phys. 12, 3336 (2010). 
${ }^{5}$ S. Luber and M. Reiher, J. Phys. Chem. A 113, 8268 (2009).

${ }^{6}$ K. H. Hopmann, K. Ruud, M. Pecul, A. Kudelski, M. Dračinský, and P. Bouř, J. Phys. Chem. B 115, 4128 (2011).

${ }^{7}$ M. Kaminski, A. Kudelski, and M. Pecul, J. Phys. Chem. B 116, 4976 (2012).

${ }^{8}$ S. Luber, J. Phys. Chem. A 117, 2760 (2013).

${ }^{9}$ A. Miani, M. S. Helfand, and S. Raugei, J. Chem. Theory Comput. 5, 2158 (2009).

${ }^{10}$ J. R. Cheeseman, M. S. Shaik, P. L. A. Popelier, and E. W. Blanch, J. Am. Chem. Soc. 133, 4991 (2011).

${ }^{11}$ J. Hudevoca, K. H. Hopmann, and P. Bour, J. Phys. Chem. B 116, 336 (2012).

${ }^{12}$ J. Kessler, M. Dračinsky, and P. Bouř, J. Comput. Chem. 34, 366 (2013).

${ }^{13}$ R. Car and M. Parrinello, Phys. Rev. Lett. 55, 2471 (1985).

${ }^{14}$ D. Marx and J. Hutter, Ab Initio Molecular Dynamics: Basic Theory and Advanced Methods (Cambridge University Press, Cambridge, 2009).

${ }^{15}$ A. Putrino and M. Parrinello, Phys. Rev. Lett. 88, 176401 (2002).

${ }^{16}$ M. Pagliai, C. Cavazzoni, G. Cardini, G. Erbacci, M. Parrinello, and V. Schettino, J. Chem. Phys. 128, 224514 (2008).

${ }^{17}$ R. D. King-Smith and R. Resta, Phys. Rev. B 47, 1651 (1993).

${ }^{18}$ R. Resta, Europhys. Lett. 22, 133 (1993).

${ }^{19}$ R. Resta, Rev. Mod. Phys. 66, 899 (1994).

${ }^{20}$ Q. Wan, L. Spanu, G. A. Galli, and F. Gygi, J. Chem. Theory Comput. 9, 4124 (2013)

${ }^{21}$ M. Thomas, M. Brehm, R. Fligg, P. Vöhringer, and B. Kirchner, Phys. Chem. Chem. Phys. 15, 6608 (2013).

${ }^{22}$ N. Marzari and D. Vanderbilt, Phys. Rev. B 56, 12847 (1997).

${ }^{23}$ P. L. Silvestrelli and M. Parrinello, Phys. Rev. Lett. 82, 3308 (1999).

${ }^{24}$ P. L. Silvestrelli and M. Parrinello, J. Chem. Phys. 111, 3572 (1999).

${ }^{25}$ B. Kirchner and J. Hutter, J. Chem. Phys. 121, 5133 (2004).

${ }^{26} \mathrm{M}$. Salanne, R. Vuilleumier, P. A. Madden, C. Simon, P. Turq, and B. Guillot, J. Phys.: Condens. Matter 20, 494207 (2008).

${ }^{27}$ X. Gonze and J.-P. Vigneron, Phys. Rev. B 39, 13120 (1989).

${ }^{28}$ X. Gonze, Phys. Rev. A 52, 1096 (1995).

${ }^{29}$ A. Putrino, D. Sebastiani, and M. Parrinello, J. Chem. Phys. 113, 7102 (2000).

${ }^{30}$ R. W. Nunes and X. Gonze, Phys. Rev. B 63, 155107 (2001).

${ }^{31}$ V. Weber, M. Iannuzzi, S. Giani, J. Hutter, R. Declerck, and M. Waroquier, J. Chem. Phys. 131, 014106 (2009).

${ }^{32}$ J. VandeVondele, M. Krack, F. Mohamed, M. Parrinello, T. Chassaing, and J. Hutter, Comput. Phys. Commun. 167, 103 (2005).

${ }^{33}$ G. Placzek, "Rayleigh-Streuung und Raman-Effekt," in Handbuch der Radiologie, edited by E. Marx (Akademische Verlagsgesellschaft, Leipzig, 1934). Vol. 6, p. 205.

${ }^{34}$ P. L. Polavarapu, L. Hecht, and L. D. Barron, J. Phys. Chem. 97, 1793 (1993).

${ }^{35}$ R. Resta, Phys. Rev. Lett. 80, 1800 (1998).

${ }^{36}$ W. Kohn and L. J. Sham, Phys. Rev. 140, 1133 (1965).

${ }^{37}$ R. M. Dreizler and E. K. U. Gross, Density Functional Theory - An Approach to the Quantum Many-Body Problem (Springer-Verlag, Berlin, 1990).

${ }^{38}$ R. G. Parr and W. Yang, Density-Functional Theory of Atoms and Molecules (Oxford University Press, Oxford, 1989).

${ }^{39} \mathrm{H}$. Eschrig, The Fundamentals of Density Functional Theory, 2nd ed. (Gutenbergplatz, Leipzig, 2003).

${ }^{40}$ K. Capelle, Braz. J. Phys. 36, 1318 (2006).

${ }^{41}$ R. M. Martin, Electronic Structure - Basic Theory and Practical Methods (Cambridge University Press, Cambridge, 2004).

${ }^{42}$ I. N. Levine, Quantum Chemistry, 5th ed. (Prentice Hall, Upper Saddle River, 2000).

${ }^{43}$ E. J. Baerends, Theor. Chem. Acc. 103, 265 (2000).

${ }^{44}$ R. G. Gordon, J. Chem. Phys. 42, 3658 (1965).

${ }^{45}$ D. A. McQuarrie, Statistical Mechanics (University Science Books, Sausalito, CA, 2000).

${ }^{46}$ R. Ramírez, T. López-Ciudad, P. Kumar, and D. Marx, J. Chem. Phys. 121, 3973 (2004).

${ }^{47} \mathrm{CP} 2 \mathrm{~K}$ developers group, URL: http://www.cp2k.org.

${ }^{48}$ A. D. Becke, Phys. Rev. A 38, 3098 (1988).

${ }^{49}$ J. P. Perdew, Phys. Rev. B 33, 8822 (1986).

${ }^{50}$ J. Neugebauer and B. A. Hess, J. Chem. Phys. 118, 7215 (2003).

${ }^{51}$ M. Reiher, G. Brehm, and S. Schneider, J. Phys. Chem. A 108, 734 (2004).
${ }^{52}$ S. Luber, C. Herrmann, and M. Reiher, J. Phys. Chem. B 112, 2218 (2008).

${ }^{53}$ S. Luber and M. Reiher, Chem. Phys. 346, 212 (2008).

${ }^{54}$ C. R. Jacob, S. Luber, and M. Reiher, ChemPhysChem 9, 2177 (2008).

${ }^{55}$ C. R. Jacob, S. Luber, and M. Reiher, J. Phys. Chem. B 113, 6558 (2009).

${ }^{56}$ C. R. Jacob, S. Luber, and M. Reiher, Chem.-Eur. J. 15, 13491 (2009).

${ }^{57}$ S. Luber, J. Neugebauer, and M. Reiher, J. Chem. Phys. 130, 064105 (2009).

${ }^{58}$ S. Luber and M. Reiher, ChemPhysChem 10, 2049 (2009).

${ }^{59}$ S. Luber and M. Reiher, J. Phys. Chem. B 114, 1057 (2010).

${ }^{60}$ O. Mohammed, S. Luber, V. S. Batista, and E. T. J. Nibbering, J. Phys. Chem. A 115, 7550 (2011).

${ }^{61}$ T. Weymuth, M. P. Haag, K. Kiewisch, S. Luber, S. Schenk, C. R. Jacob, C. Herrmann, J. Neugebauer, and M. Reiher, J. Comput. Chem. 33, 2186 (2012).

${ }^{62}$ S. Luber, K. Adamczyk, E. T. J. Nibbering, and V. S. Batista, J. Phys. Chem. A 117, 5269 (2013).

${ }^{63}$ B. Varnholt, P. Oulevey, S. Luber, C. Kumara, A. Dass, and T. Bürgi, J. Phys. Chem. C 118, 9604 (2014).

${ }^{64}$ C. Lee, W. Yang, and R. G. Parr, Phys. Rev. B 37, 785 (1988).

${ }^{65}$ P. L. Silvestrelli, M. Bernasconi, and M. Parrinello, Chem. Phys. Lett. 277, 478 (1997).

${ }^{66}$ M.-P. Gaigeot and M. Sprik, J. Phys. Chem. B 107, 10344 (2003).

${ }^{67}$ J.-W. Handgraaf, E. J. Meijer, and M.-P. Gaigeot, J. Chem. Phys. 21, 10111 (2004).

${ }^{68}$ M.-P. Gaigeot, R. Vuilleumier, M. Sprik, and D. Borgis, J. Chem. Theory Comput. 1, 772 (2005).

${ }^{69}$ D. C. Marinica, G. Greǵoir, C. Desfrançois, J. P. Schermann, D. Borgis, and M. P. Gaigeot, J. Phys. Chem. A 110, 8802 (2006).

${ }^{70}$ M.-P. Gaigeot, Phys. Chem. Chem. Phys. 12, 10198 (2010).

${ }^{71}$ A. Cimas, T. D. Vaden, T. S. J. A. de Boer, L. C. Snoek, and M.-P. Gaigeot, J. Chem. Theory Comput. 5, 1068 (2009).

${ }^{72}$ A. Cimas, P. Maitre, G. Ohanessian, and M.-P. Gaigeot, J. Chem. Theory Comput. 5, 2388 (2009).

${ }^{73}$ J. P. Perdew, K. Burke, and M. Ernzerhof, Phys. Rev. Lett. 77, 3865 (1996).

${ }^{74}$ J. P. Perdew, K. Burke, and M. Ernzerhof, Phys. Rev. Lett. 78, 1396 (1997).

${ }^{75}$ S. Goedecker, M. Teter, and J. Hutter, Phys. Rev. B 54, 1703 (1996).

${ }^{76}$ C. Hartwigsen, S. Goedecker, and J. Hutter, Phys. Rev. B 58, 3641 (1998).

${ }^{77}$ M. Krack, Theor. Chem. Acc. 114, 145 (2005).

${ }^{78}$ S. Grimme, J. Antony, S. Ehrlich, and H. Krieg, J. Chem. Phys. 132, 154104 (2010).

${ }^{79}$ D. R. Lide, CRC Handbook of Chemistry and Physics, 90th ed. (CRC Press, Cleveland, Ohio, 2009).

${ }^{80}$ S. Nosé, J. Chem. Phys. 81, 511 (1984).

${ }^{81}$ S. Nosé, Mol. Phys. 52, 255 (1984).

${ }^{82}$ M. Brehm and B. Kirchner, J. Chem. Inf. Model. 51, 2007 (2011).

${ }^{83}$ R. Ahlrichs, M. Bär, M. Häser, H. Horn, and C. Kölmel, Chem. Phys. Lett. 162, 165 (1989).

${ }^{84}$ W. G. Bickley, Math. Gaz. 25, 19 (1941).

${ }^{85}$ F. Weigend and R. Ahlrichs, Phys. Chem. Chem. Phys. 7, 3297 (2005).

${ }^{86}$ F. Weigend, Phys. Chem. Chem. Phys. 8, 1057 (2006).

${ }^{87}$ M. E. Casida, "Time-dependent density functional response theory for molecules," in Recent Advances in Density Functional Methods, edited by D. P. Chong (World Scientific, Singapore, 1995), Vol. 1, pp. 155-192.

${ }^{88}$ R. Bauernschmitt, M. Häser, O. Treutler, and R. Ahlrichs, Chem. Phys. Lett. 264, 573 (1997).

${ }^{89}$ L. D. Barron and P. L. Polavarapu, Mol. Phys. 65, 659 (1988).

${ }^{90}$ M. Carnell, S. Peyerimhoff, A. Breest, K. Gödderz, P. Ochmann, and J. Hermes, Chem. Phys. Lett. 180, 477 (1991).

${ }^{91}$ P. K. Bose, P. L. Polavarapu, L. D. Barron, and L. D. Hecht, J. Phys. Chem. 94, 1734 (1990).

${ }^{92}$ M. C. Tam, N. J. Russ, and T. D. Crawford, J. Chem. Phys. 121(8), 35503557 (2004).

${ }^{93}$ M. Reiher, V. Liégeois, and K. Ruud, J. Phys. Chem. A 109, 7567 (2005).

${ }^{94}$ J. Šebestík and P. Bouř, J. Phys. Chem. Lett. 2, 498 (2011).

${ }^{95}$ J. Bloino and V. Barone, J. Chem. Phys. 136, 124108 (2012).

${ }^{96}$ See supplementary material at http://dx.doi.org/10.1063/1.4894425 for the spectra.

${ }^{97}$ M. Sprik, J. Hutter, and M. Parrinello, J. Chem. Phys. 105, 1142 (1996).

${ }^{98}$ C. Adamo and V. Barone, J. Chem. Phys. 110, 6158 (1999).

${ }^{99}$ C. Zhang, D. Donadio, F. Gygi, and G. Galli, J. Chem. Theory Comput. 7, 1443 (2011). 